\title{
1579 NM FIBER LASER SOURCE FOR SPACEBORNE MONITORING OF CARBON DIOXIDE
}

\author{
Nicolas Cézard*1 ${ }^{*}$ Laurent Lombard, Julien Le Gouët, Didier Goular, Alexandre Bresson, \\ Agnès Dolfi-Bouteyre, Guillaume Canat
}

${ }^{I}$ ONERA - The French Aerospace Lab - F-91761 Palaiseau, France,

*Email:nicolas.cezard@onera.fr

\begin{abstract}
We report on the development of a $1579 \mathrm{~nm}$ pulsed fiber laser source with high peak-power, intended to be used as a lidar source for $\mathrm{CO}_{2}$ monitoring from space. We first discuss watervapor sensitivity of spaceborne $\mathrm{CO}_{2}$ measurements by lidar and point the interest of the $1579 \mathrm{~nm}$ wavelength with that respect. Then we detail the current development status of the pulsed fiber laser source.
\end{abstract}

\section{INTRODUCTION}

Integrated-Path Differential Absorption Lidar (IPDA) has been proposed for next-generation spaceborne $\mathrm{CO}_{2}$ monitoring systems [1]. Two wavelengths windows, at $2 \mu \mathrm{m}$ and $1.5 \mu \mathrm{m}$, were identified as suitable for this task. We will focus here on the $1.5 \mu \mathrm{m}$ band, which shows a less favorable weighting function in the troposphere but benefits of more mature detector technologies. A detailed multi-criteria wavelength selection was conducted in early studies that led to identify the $1572 \mathrm{~nm} \mathrm{CO}$ absorption transition as a good candidate [1]. Several laser transmitters have been proposed to address this wavelength and fulfill all the desired requirements for space operation. An OPObased pulsed transmitter has been developed through A-SCOPE project [2], while fiber-based pulsed lasers are developed for ASCENDS[3-4]. A fiber-based, frequency modulated cw laser has also been proposed at $1573 \mathrm{~nm}$ [5].

In 2009, it was suggested by Caron and Durand [6] that using the $\mathrm{CO}_{2}$ absorption transition at $1579 \mathrm{~nm}$ could dramatically reduce the measurement sensitivity to water vapor uncertainties. ONERA was contracted in 2011 by ESA to develop a high energy pulsed fiber laser emitting around $1579 \mathrm{~nm}$, through HEPILAS project. In this paper, we first recall how water-vapor sensitivity can be evaluated for a spaceborne $\mathrm{CO}_{2}$ IPDA system, and we confirm, with detailed conditions, that this sensitivity can be minimized by an appropriate choice of ON and OFF wavelengths around the $1579 \mathrm{~nm}$ transition. Then we report on the current status of the $1579 \mathrm{~nm}$ pulsed fiber laser source under development in HEPILAS project.

\section{WATER-VAPOR SENSITIVITY OF A $\mathrm{CO}_{2}$ IPDA LIDAR AT 1579 NM}

The IPDA lidar aims at measuring the $\mathrm{CO}_{2}$ column-integrated dry air volume mixing ratio, noted $\rho_{\mathrm{CO} 2}$. This quantity can be estimated from the measured Differential Absorption Optical Depth (DAOD) noted $\Delta \tau_{\text {measured }}=\tau\left(\lambda_{\mathrm{ON}}\right)-\tau\left(\lambda_{\mathrm{OFF}}\right)$, using the following relationship:

$$
\rho_{\mathrm{CO}_{2}}=\frac{1}{I W F_{\text {assumed }}}\left[\Delta \tau_{\text {measured }}-\Delta \tau_{\mathrm{H}_{2} \mathrm{O}_{-} \text {assumed }}\right]
$$

The retrieval thus requires ancillary assumptions concerning values of the water-vapor DAOD and of the so-called Integrated Weighting Function (IWF) defined as:

$$
I W F_{\text {assumed }}=\int_{0}^{P_{G}} \frac{\Delta \sigma_{\mathrm{CO}_{2}}(P)}{g m_{\text {dry }}\left(1+k \rho_{\mathrm{H}_{2} \mathrm{O}_{-} \text {assumed }}(P)\right)} d P
$$

Where $\mathrm{P}_{\mathrm{G}}$ is the ground pressure, $\Delta \sigma_{\mathrm{CO} 2}(\mathrm{P})$ is the pressure-dependent difference between $\mathrm{ON}$-line and OFF-line absorption cross-sections (in $\mathrm{m}^{2}$ ), $\mathrm{g}=9.8 \mathrm{~m} / \mathrm{s}^{2}$ is the Earth gravity, $\mathrm{k}=\mathrm{m}_{\mathrm{dry}} / \mathrm{m}_{\mathrm{H} 2 \mathrm{O}}$ is a constant ratio, $\mathrm{m}_{\mathrm{dry}}$ and $\mathrm{m}_{\mathrm{H} 2 \mathrm{O}}$ are the average masses of one molecule of dry air and water vapor respectively, and $\rho_{\text {H2O_assumed }}(\mathrm{P})$ is the 
assumed vertical profile of water vapor dry air volume mixing ratio.

Any error concerning the water vapor profile induces errors on both IWF and $\Delta \tau_{\mathrm{H} 2 \mathrm{O}}$, that eventually translate into a Relative Systematic Error (RSE) on the retrieved value of $\rho_{\mathrm{CO} 2}$. However, as pointed out by Caron and Durand [6], biases induced by both terms have opposite signs and can mutually cancel under favorable conditions. A useful figure of merit for watervapor sensitivity can be obtained assuming that the water vapor profile is known up to a scaling constant $(1+\varepsilon)$, that is :

$$
\rho_{\mathrm{H}_{2} \mathrm{O}_{-} \text {assumed }}(P)=(1+\varepsilon) \rho_{\mathrm{H}_{2} \mathrm{O}_{-} \text {real }}(P)
$$

Following Eqs. 18-22 in ref.6, it can then be shown that the RSE induced on $\rho_{\mathrm{CO} 2}$ by an $\varepsilon$ error on the water vapor profile reads:

$$
R S E=-\frac{\varepsilon}{\Delta \tau_{C O_{2}}} \int_{0}^{P_{G}} \frac{\rho_{\mathrm{H}_{2} \mathrm{O}}(P)\left(\Delta \sigma_{\mathrm{H}_{2} \mathrm{O}}(P)-k \rho_{\mathrm{CO}_{2}} \Delta \sigma_{\mathrm{CO}_{2}}(P)\right)}{g m_{d r y}\left[1+k \rho_{\mathrm{H}_{2} \mathrm{O}}(P)\right]^{2}} d P
$$

This induced RSE is a bi-dimensional function of ON and OFF wavelengths. It can cancel if the water vapor DAOD is slightly positive (not null), as can be seen from the right hand side term's numerator. Figure 1 shows the RSE (expressed in $\mathrm{dB}$ ) induced on $\rho_{\mathrm{CO} 2}$ by an $\varepsilon=5 \%$ error on the water vapor vertical profile, as a function of ON-line and OFF-line frequencies (expressed as frequencies relative to the $1579 \mathrm{~nm}$ line center). For a worst-case assessment of humidity sensitivity, we have assumed a tropical profile for $\mathrm{H}_{2} \mathrm{O}$, with a $20 \mathrm{~g} / \mathrm{kg}$ specific humidity on ground. A constant $380 \mathrm{ppm}$ profile was assumed for $\mathrm{CO}_{2}$. Voigt lineshapes and HITRAN 2008 database were used to compute $\mathrm{H}_{2} \mathrm{O}$ and $\mathrm{CO}_{2}$ cross sections.

Adapting Table.1 of ref.2 to the current wavelength, the threshold RSE (maximum tolerable) and target RSE (scientific objective) are computed to be approximately $0.02 \%(-37$ $\mathrm{dB})$ and $0.006 \%(-42 \mathrm{~dB})$ respectively. Figure 1 clearly shows a line were the water-vaporinduced RSE is virtually cancelled (below -60 $\mathrm{dB})$. For example, if the ON-line wavelength is chosen to be $+0.7 \mathrm{GHz}$, then the OFF-line wavelength should be tuned at $-11.3 \mathrm{GHz}$ (red point on figure 1). Though not plotted here, it can also be shown that the RSE cannot be cancelled if the ON-line frequency is chosen negative with respect to the line center.

However, beyond the water-vapor insensitivity criterion, selecting the lidar ON-line wavelength requires trading off many other figures of merit. Indeed, measurement RSE can also be induced by errors on the temperature profile, ground pressure, ground altitude, laser spectral stability, spectral linewidth, spectral purity...The Relative Random Errors (RRE) and the allowed RRE \& RSE budgets are also ON-line-wavelengthdependent. But if a positive ON-line frequency can be selected as a good tradeoff, then an OFFline wavelength can also be found so that the water-vapor induced RSE is cancelled or at least minimized. To our knowledge, this possibility was not numerically assessed in the literature so far, and such a behavior is not found in the vicinity of the A-SCOPE transition at $1572 \mathrm{~nm}$. This justifies the interest of the $1579 \mathrm{~nm}$ wavelength for a spaceborne IPDA $\mathrm{CO}_{2}$ lidar.

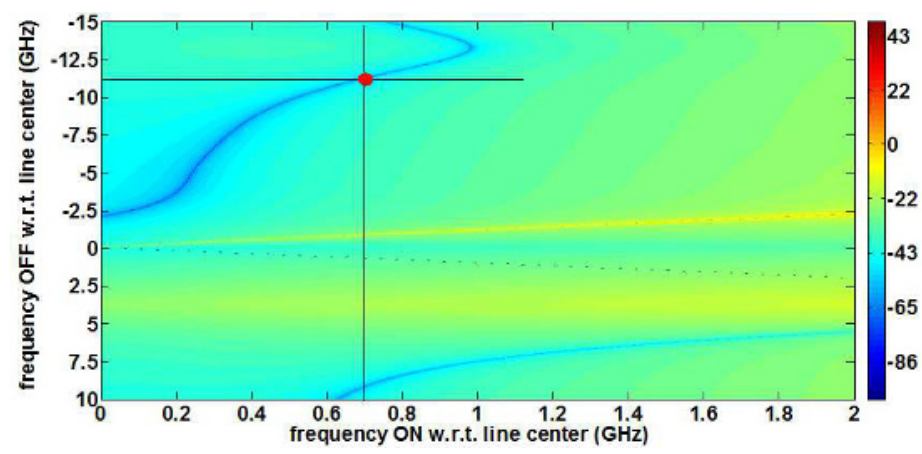

Figure 1: RSE in $\mathrm{dB}$ arising from a $5 \%$ error on a tropical water-vapor profile $(20 \mathrm{~g} / \mathrm{kg}$ at ground). The blue line in the up-left corner indicates a line of virtually cancelled water vapor sensitivity (very low induced RSE). 


\section{HIGH ENERGY PULSED FIBER LASER SOURCE AT 1579 NM}

A high energy, pulsed fiber laser source at $1579 \mathrm{~nm}$ is under development at ONERA through ESA-funded HEPILAS project. The expected performances are the following: pulse energy $260 \mu \mathrm{J}$, pulse duration $150 \mathrm{~ns}$, beam quality $\mathrm{M}^{2}<2$, pulse linewidth $<60 \mathrm{MHz}$, laser stability $200 \mathrm{kHz}$ over 10 seconds.

Laser amplification is challenging here because i) Erbium gain is lower at $1579 \mathrm{~nm}$ than at the more 'classical' $1572 \mathrm{~nm}$ wavelength, and ii) since short pulses are demanded, the Stimulated Brillouin Scattering (SBS) threshold peak power is quickly reached. It is worth noticing, for comparison, that the $1572 \mathrm{~nm}$ fiber laser under development for ASCENDS project [4] delivers much longer pulses $(1.5 \mu \mathrm{s})$.

The HEPILAS laser system architecture is a three stage master oscillator power amplifier (MOPA) seeded by tunable and well-referenced oscillators. The seed lasers are Telecom DFB laser diodes that are stabilized by a master/slave architecture. At the output of the stabilized oscillator, $2 \mathrm{~mW}$ peak power with low noise are available with a time pattern composed of ' $\mathrm{ON}$ ' and 'OFF' pulses controlled by an optical switch. The pattern pulse repetition frequency is $2 \mathrm{kHz}$.

We measured the short term stability of the beatnote of two identical free running seeders using a high speed counter. An Allan standard deviation of $\sim 220 \mathrm{kHz}$ over $10 \mathrm{~s}$ was measured for the beatnote. Assuming identical stability for both diodes, a stability of $155 \mathrm{kHz}$ over $10 \mathrm{~s}$ can be attributed to each DFB seeder. For stable spaceborne operation, it is planned to lock a master oscillator at the center of the $1579 \mathrm{~nm}$ transition using a $\mathrm{CO}_{2}$ multipass absorption cell and a modulation/lock-in technique, and then reference ON-line and OFF-line oscillators to the master using offset beatnote stabilization techniques. We also measured the spectral purity of seed lasers using a high resolution OSA (for broadband measurement) and heterodyning on an electrical spectrum analyzer (for high resolution measurement). A single longitudinal mode behavior with $50 \mathrm{~dB}$ of Side-Mode Suppression Ratio and a spectral purity better than $99.95 \%$ within $1 \mathrm{GHz}$ were found. These parameters meet requirements for spaceborne operation.

Starting from a $2 \mathrm{~mW}$ peak power seed, the objective is to reach about $2 \mathrm{~kW}$ peak power at the exit of a three-stage amplification chain. However, amplification of single frequency DFB lasers at high peak power leads to Stimulated Brillouin Scattering. The threshold for this nonlinear effect is of the order of $80 \mathrm{~W}$ per meter of fiber in standard single-mode fibers. Therefore, in order to reach the desired high peak power at the third stage output, we considered simultaneous implementation of various well-known SBS mitigation techniques, such as the use of large mode area fibers, composition tuning, and strain gradient generation along the fiber.

We have built a preliminary version of the fiber laser source, sketched in figure 2 .

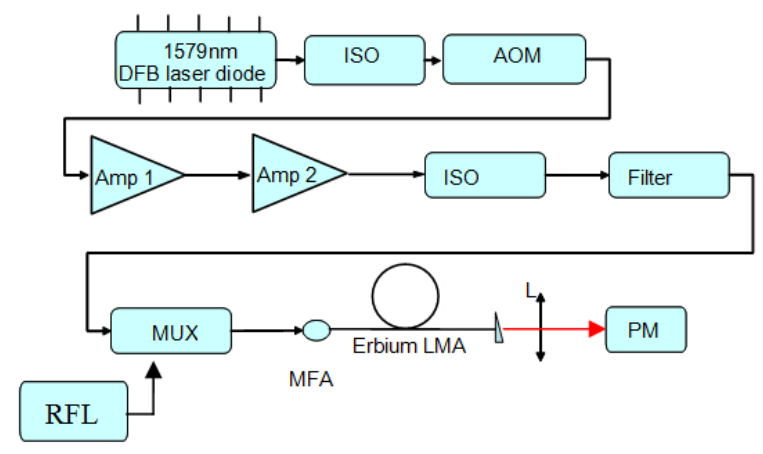

Figure 2: Preliminary setup of HEPILAS fiber laser source

A $30 \mathrm{~mW}$ single frequency $1579 \mathrm{~nm}$ DFB laser diode is connected, after optical isolation (ISO), to an Acousto-Optic Modulator (AOM) used to generate gaussian pulse shape with pulse duration $150 \mathrm{~ns}$ at $4 \mathrm{kHz}$ pulse repetition frequency. The pulses enter a double pass preamplifier (Amp 1) built using a standard single-mode L-band fiber core-pumped at $1480 \mathrm{~nm}$. The amplified pulses enter a second stage of amplification (Amp 2), built using a $12 \mu \mathrm{m}$-core Erbium-doped fiber, core-pumped at $1480 \mathrm{~nm}$. After isolation and spectral filtering to remove the Amplified Spontaneous Emission (ASE), $31 \mathrm{~W}$ peak power pulses enter in the 
third power amplifier stage. The latter is built using a $40 \mu \mathrm{m}$-core nLight Er40-60 Large Mode Area (LMA) fiber. It is core-pumped in copropagating configuration by a $4 \mathrm{~W}$ Raman Fiber Laser (RFL) (through a single-mode wavelength multiplexer (MUX) and a Mode Field Adaptation (MFA)). The peak power is monitored by a power-meter (PM) at the third stage exit.

Using this setup, the peak power was found to be quickly limited by SBS to $550 \mathrm{~W}$. We then coiled the fiber with a triangular strain distribution. It is indeed known that the central frequency of the Brillouin gain curve can be shifted by applying strain. Applying an optimal strain distribution for a given signal distribution in the fiber will result in a flat Brillouin spectrum, and consequently, an increased SBS threshold peak power. We have shown that a triangular distribution can be used in this case. With this improvement, we have obtained up to $260 \mu \mathrm{J}$ per pulse, corresponding to $1.7 \mathrm{~kW}$ peak power. The peak power was thus increased by a factor almost 4 using fiber strain distribution. The slope efficiency reaches $23 \%$. So far the output $1579 \mathrm{~nm}$ peak power is only limited by the available pump power. There was some concern that strain could modify the mode repartition in the LMA fiber, but we measured an excellent beam quality with $\mathrm{M}^{2}<1.2$.

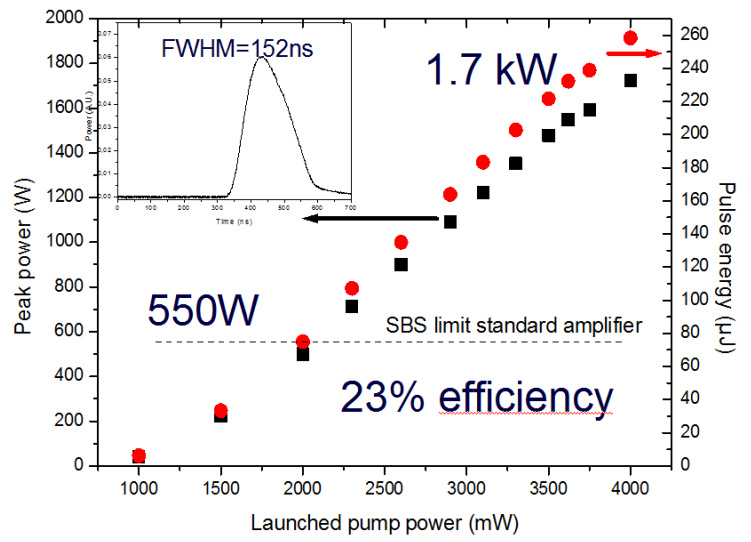

Fig. 3. Peak power and pulse energy at the exit of the third amplification stage, as a function of pump power. A triangular strain profile is applied to the LMA fiber, allowing overcoming the $550 \mathrm{~W}$ limit (obtained without strain).

\section{CONCLUSIONS}

For $\mathrm{CO}_{2}$ monitoring by a spaceborne IPDA Lidar, working around $1579 \mathrm{~nm}$ could offer a very low sensitivity to water-vapor profile errors, with an appropriate choice of ON-line and OFF-line wavelengths. It can thus be seen as an interesting alternative to the initially selected $1572 \mathrm{~nm}$ wavelength. A fiber laser is under development at ONERA to further assess this possibility. Though the $1579 \mathrm{~nm}$ wavelength is less favorable than $1572 \mathrm{~nm}$ for Erbium-doped fiber amplification, we have obtained $1.7 \mathrm{~kW}$ peak power for $150 \mathrm{~ns}$ pulses (pulse energy $260 \mu \mathrm{J})$, using SBS mitigation techniques (especially strain distribution). Further work will focus on increasing the peak power further, and assessing strained-fiber lifetime and radiation handling capabilities.

\section{ACKNOWLEDGEMENT}

The fiber laser development is funded by the European Space Agency under HEPILAS project (contract \#4000104022/11/NL/CP).

\section{REFERENCES}

[1] G.Ehret et al, Appl. Phys.B 90, 593-608 (2008)

[2] J.Caron et al, SPIE Proc. 7479, 74790E, 1-15 (2009)

[3] K.Numata et al, Appl.Opt. 50, 1047-1056 (2011)

[4] W. Lu et al, SPIE Proc. 8961, 89610C, 1-10 (2014)

[5] S.Kameyama et al. Opt. Lett. 34, 1513-1515 (2009)

[6] J.Caron et Y.Durand, Appl.Opt. 48, 54135422 (2009) 